\title{
Reversible Interacting-Particle Reaction Dynamics
}

\author{
Christoph Fröhner* and Frank Noé ${ }^{*}$ \\ Freie Universität Berlin, Fachbereich Mathematik und Informatik, \\ Arnimallee 6, 14195 Berlin, Germany
}

(Dated: July 20, 2018)

\begin{abstract}
Interacting-Particle Reaction Dynamics (iPRD) simulates the spatiotemporal evolution of particles that experience interaction forces and can react with one another. The combination of interaction forces and reactions enable a wide range of complex reactive systems in biology and chemistry, but give rise to new questions such as how to evolve the dynamical equations in a computationally efficient and statistically correct manner. Here we consider reversible reactions such as $\mathrm{A}+\mathrm{B} \rightleftarrows \mathrm{C}$ with interacting particles and derive expressions for the microscopic iPRD simulation parameters such that desired values for the equilibrium constant and the dissociation rate are obtained in the dilute limit. We then introduce a Monte-Carlo algorithm that ensures detailed balance in the iPRD time-evolution (iPRD-DB). iPRD-DB guarantees the correct thermodynamics at all concentrations and maintains the desired kinetics in the dilute limit, where chemical rates are well-defined and kinetic measurement experiments usually operate. We show that in dense particle systems, the incorporation of detailed balance is essential to obtain physically realistic solutions. iPRD-DB is implemented in ReaDDy 2 (https://readdy.github.io).
\end{abstract}

Keywords: Reaction Kinetics, Reaction-Diffusion dynamics, interacting-Particle Reaction Dynamics, ParticleBased Reaction-Diffusion, Detailed Balance, Monte Carlo, Statistical Physics

\footnotetext{
* christoph.froehner@fu-berlin.de

$\dagger$ corresponding author: frank.noe@fu-berlin.de
} 


\section{INTRODUCTION}

Particle based reaction diffusion (PBRD) dynamics is a detailed model for simulating the spatiotemporal evolution of reactive particles [1-4]. Resolving the trajectories of every reactive particle is important in applications where the reactants cannot be assumed to be spatially well-mixed [5,6] or always sufficiently abundant to be described by a continuous concentration $[7,8]$ - e.g., consider many cases of cellular signalling and reactions in nontrivial architectures [9-11]. A common implementation of PBRD is to propagate particle positions with overdamped Langevin dynamics (Brownian motion) in discrete time steps, and execute discrete reaction events such as $\mathrm{A}+\mathrm{B} \rightarrow \mathrm{C}$ with a certain probability when two reactive particles $\mathrm{A}$ and $\mathrm{B}$ are close in space. When the system is sufficiently dilute, such simulations can be sped up by exploiting solutions of the one- or two-particle diffusion equation [2, 12-15].

A recent extension of PBRD is the interacting-Particle Reaction Dynamics (iPRD) method [16-18], in which particles are additionally subject to interaction forces. Alternatively, iPRD could be characterized as a form of coarse-grained Molecular Dynamics (MD) simulation with reactions between particles. Particle interaction forces are useful to model order and structure on mesoscopic lengthscales, such as the space-exclusion in dense particle systems [16, 19], the restriction of diffusing particles to arbitrarily-shaped membranes [16, 20, 21], the large-scale structure of polymers [22] and membranes [23], and the clustering of attractive proteins [24]. The combination of interaction forces and reactions allow an even wider range of complex reactive systems in biology and chemistry to be modeled, such as the dynamics of phototransduction that involve protein diffusion in particle-dense photoreceptor membranes [21], the effect of transmembrane protein oligomers on these dynamics [20], the recruitment of proteins to endosomes [25, 26], and the assembly, diffusion, and dissociation of polymers [22]. The idea of combining PBRD with particle interaction forces is also found in MD-GFRD [27, 28], where the close particle interactions are simulated by MD and the reaction-diffusion model is used to derive an efficient way to propagate particles while they are not-interacting. In contrast, in iPRD particle interactions and reactions occur simultaneously, with the idea that reaction events are a suitable way to coarse-grain complicated events such as protein-protein binding, whose kinetics might be obtained from Markov State Models of all-atom MD simulations [29]. MD-GFRD simulations can be used to speed up iPRD simulations when the system is sufficiently dilute [27, 28, 30], and with free-propagator reweighting, this speedup can also be obtained in the regime where particles are interacting [31].

An open question is: What is the statistically correct way to model the dynamical evolution of simultaneously interacting and reacting particles? Specifically, we consider reversible reactions, such as $\mathrm{A}+\mathrm{B} \rightleftarrows \mathrm{C}$, as they are found in nature, but also in technological applications. Examples include reversible protein-drug binding [32, 33], reversible proteinprotein association that can now be simulated at atomistic detail [34], and metal ion deposition to / removal from electrodes in batteries that are driving charging and discharging $[35,36]$. To derive a statistically correct simulation scheme of $\mathrm{A}+\mathrm{B} \rightleftarrows \mathrm{C}$ via iPRD, we need to answer the following questions:

1. Which bimolecular reaction scheme should be used, i.e. under which conditions will two particles $\mathrm{A}$ and $\mathrm{B}$ fuse into a $\mathrm{C}$ particle?

2. How do we choose the microscopic parameters of this reaction scheme such that the iPRD simulation samples the macroscopic kinetic quantities that have been obtained from experiments or more detailed MD simulations? 
3. When executing $\mathrm{A}+\mathrm{B} \rightarrow \mathrm{C}$ or $\mathrm{C} \rightarrow \mathrm{A}+\mathrm{B}$, where should the product particles be placed, such that the simulation obeys detailed balance?

The answers to these three questions are coupled.

Question 1: For the sake of analytical computations, the best-studied reaction scheme is the Smoluchowski model where diffusing particles react instantly when they establish contact, defined by a reaction distance $R$ [37]. The Collins-Kimball model [38] reduces the probability of reacting upon contact to a finite value $\leq 1$. Reversible reactions in the Collins-Kimball model are discussed in [39], for interacting particles of isolated pairs an analytical description is found in [40]. In iPRD simulations we instead use the Doi model [41, 42]:

$$
\mathrm{A}+\mathrm{B} \rightleftarrows \mathrm{AB} \rightleftarrows \mathrm{C}
$$

Here two particles $\mathrm{A}$ and $\mathrm{B}$ form a reactive complex $\mathrm{AB}$ when their distance is less or equal to $R$. This process is simulated by the dynamical model that propagates particles (e.g. overdamped Langevin equation). Whenever a reactive complex $\mathrm{AB}$ exists, it can decay to a $\mathrm{C}$ particle with a microscopic rate constant $\lambda$. The reverse process happens with a microscopic rate constant $k_{\text {off }}$. The Doi model is well compatible with a finite-time-stepping simulation scheme, where the formation of $\mathrm{AB}$ can be easily checked in every time-step as part of the particle neighbor list update.

Question 2: When using the Doi model, how should the parameters in this model be chosen? The dissociation rate constant $k_{\text {off }}$ can be directly obtained from kinetic experiments or all-atom MD simulations with accelerated sampling methods [33, 34, 43]. For the Doi model where $\mathrm{A}$ and $\mathrm{B}$ encounter from a long distance via normal diffusion without interaction forces, the association parameters $R$ and $\lambda$ can be computed from an equation derived in $[1,44]$. When A and B interact, such a result can still be obtained numerically [45]. In Sec. II we develop a theory for the $\mathrm{A}+\mathrm{B} \rightleftarrows \mathrm{C}$ reaction of an isolated pair, that is independent of the diffusion coefficient $D$. This enables to choose $\lambda$ for given dissociation rate constant $k_{\text {off }}$, interaction radius $R$, and $\mathrm{A}-\mathrm{B}$ interaction potential such that the iPRD simulation will produce a desired equilibrium constant and association rate constant at low particle concentrations, as they are typically found in experiments measuring these constants.

Question 3: Time-reversible processes evolving in thermodynamic equilibrium obey detailed balance [46]. For example, consider that we have system with one particle A and B each at positions $\mathbf{x}_{A}, \mathbf{x}_{B}$ and we perform the forward reaction to a system with one particle $\mathrm{C}$ at position $\mathbf{x}_{C}$. Detailed balance implies that the equilibrium probability of being in the $\mathrm{A}, \mathrm{B}$ system at $\mathbf{x}_{A}, \mathbf{x}_{B}$ times the forward reaction rate must be equal to the equilibrium probability of being in the $\mathrm{C}$ system at $\mathbf{x}_{C}$ times the backward reaction rate, and this must be true for all system configurations. Vice versa, enforcing detailed balance is a technically convenient way to automatically achieve a desired equilibrium distribution. It implies a relationship between forward and backward reaction rates and also that the reaction scheme that allows for a forward reaction $\mathbf{x}_{A}, \mathbf{x}_{B} \rightarrow \mathbf{x}_{C}$ must also allow for the reverse reaction, and vice versa. For non-interacting PBRD, a detailed balance scheme was first introduced in [47]. Other schemes have been developed more recently $[48,49]$. In Sec. III, we develop a general detailed-balance scheme for iPRD (iPRD-DB). The scheme includes a Metropolis-Hastings [50, 51] acceptance step that ensures the resulting dynamics fulfill detailed balance for abitrary configurations of interacting particles. In the dilute limit (one A and B particle pair reacting to a single $\mathrm{C}$ particle and back), the proposal steps are designed such that they are always accepted and the desired equilibrium association and dissociation rate constants are obtained. When the so-parametrized particles enter a dense phase, the kinetics and equilibria will naturally change, but do so in a physically realistic manner. In particular, we show that in a dense particle system where the reaction $\mathrm{A}+\mathrm{B} \rightleftarrows \mathrm{C}$ involves a change in effective particle volume, 
the iPRD-DB scheme leads to a solution that is consistent with Le Chatelier's principle, while a regular Doi scheme that ignores detailed balance produces unphysical solutions.

The implementation of the iPRD-DB scheme is included in the ReaDDy 2 software package [22].

\section{BIMOLECULAR REACTION IN EQUILIBRIUM}

We consider a system of molecules with three species, in which molecules $\mathrm{A}$ and $\mathrm{B}$ reversibly form a complex C. We want to simulate particle dynamics involving such reactions with iPRD, where particles interact with a potential when they are close, and a certain microscopic reaction scheme is employed, see Fig. 1. This section answers the question how the microscopic parameters of this reaction scheme need to be chosen such that the equilibrium constant and the dissociation rate measurable in a bulk experiments will be reproduced. This result will be used in the next section as part of designing a scheme obeying detailed balance.

\section{A. Macroscopic rate model}

The macroscopic reaction dynamics is described by the scheme

$$
\mathrm{A}+\mathrm{B} \underset{k_{\text {off }}}{\stackrel{k_{\text {on }}}{\rightleftarrows}} \mathrm{C}
$$

where $k_{\text {on }}$ is a macroscopic bimolecular association rate constant, measured in units of per time and per concentration, while $k_{\text {off }}$ is the dissociation rate constant, measured in units of per time. These are related to the macroscopic dissociation constant $K_{d}$, measured in units of concentration:

$$
K_{d}=\frac{k_{\mathrm{off}}}{k_{\mathrm{on}}} .
$$

We assume that both the association- and the dissociation process obey a linear rate law [52], according to the law of mass action (LMA). We define the effective association rate $K_{\text {on }}$

$$
K_{\mathrm{on}}=k_{\mathrm{on}} V^{-1}
$$

which is the frequency of association per $\mathrm{AB}$ complex. Likewise we define the effective dissociation rate $K_{\text {off }}$

$$
K_{\mathrm{off}}=k_{\mathrm{off}},
$$

which is the frequency of dissociation per $\mathrm{C}$ molecule. We denote $\pi_{i}$ as the stationary probability of state $i$. The ratio of stationary probabilities $\pi_{A B} / \pi_{C}$ is given by the ratio of effective rates in equilibrium, where the number of association events per time is equal to the number of dissociation events per time

$$
\frac{\pi_{A B}}{\pi_{C}}=\frac{K_{\mathrm{off}}}{K_{\mathrm{on}}}=\frac{[A]_{\mathrm{eq}}[B]_{\mathrm{eq}}}{[C]_{\mathrm{eq}}} V=K_{d} V .
$$




\section{B. Microscopic distribution}

For the following we will assume that there is only either one pair of A and B particles or one $\mathrm{C}$ particle which live inside the volume $V$. The vectors $\mathbf{x} \in \mathbb{R}^{9}$, contain the euclidean positions for three particles. Individual positions are denoted by $\mathbf{x}_{a}, \mathbf{x}_{b}$, and $\mathbf{x}_{c}$ for particles $\mathrm{A}, \mathrm{B}$, and $\mathrm{C}$ respectively. Additionally there is a phase $i \in\{A B, C\}$, where $A B$ is the dissociated phase and $C$ is the associated phase. The joint distribution for states $x_{i}=(\mathbf{x}, i) \in \mathbb{R}^{9} \times\{A B, C\}$ of finding the system in phase $i$ and particle positions $\mathbf{x}$ is

$$
p\left(x_{i}\right)= \begin{cases}\pi_{A B} p_{A B}(\mathbf{x}) & \text { for } i=A B \\ \pi_{C} p_{C}(\mathbf{x}) & \text { for } i=C\end{cases}
$$

Note that in phase $A B$ there is still a position for the $\mathrm{C}$ particle, such that the dimension of the microscopic phase space is equal for both phases. The same occurs for the positions of $\mathrm{A}$ and $\mathrm{B}$ in the phase $C$. In both cases, the residual variables have no effect. In phase space integrals these will be accounted for by a volume factor. Hence all phase space integrals use the measure $\mathrm{d} \mathbf{x}=\mathrm{d} \mathbf{x}_{a} \mathrm{~d} \mathbf{x}_{b} \mathrm{~d} \mathbf{x}_{c}$, where each $\mathrm{d} \mathbf{x}_{j}$ has units of volume. Introducing a Fock space for treatment of changing number of particles is circumvented by considering at most three particles - the isolated pair and the complex - and having the non existing particles contribute a constant factor to the partition function.

In phase $A B$ the two particles $\mathrm{A}$ and $\mathrm{B}$ are subject to an interaction potential $U(\mathbf{x})=$ $U\left(\left|\mathbf{x}_{b}-\mathbf{x}_{a}\right|\right)=U(r)$ depending only on the distance $r=\left|\mathbf{x}_{b}-\mathbf{x}_{a}\right|$ of A and B. The potential is cut off at $R_{\text {int }}$, i.e. $U(r)=0$, if $r>R_{\text {int }}$. The stationary distribution of positions $\mathbf{x}$ in phase $A B$ is

$$
p_{A B}(\mathbf{x})=Z_{A B}^{-1} \exp (-\beta U(r)) \text { with } r=\left|\mathbf{x}_{b}-\mathbf{x}_{a}\right|
$$

where $\beta^{-1}=k_{B} T$ is the thermal energy of the system which is coupled to a heat bath with temperature Tand the normalization constant can be computed as follows (see Appendix A),

$$
\begin{aligned}
Z_{A B} & =V^{2}\left(V-V_{\mathrm{ex}}\right) \\
V_{\mathrm{ex}} & =V_{\mathrm{int}}-V_{\mathrm{int}}^{\mathrm{eff}} \\
V_{\mathrm{int}} & =\frac{4}{3} \pi R_{\mathrm{int}}^{3} \\
V_{\mathrm{int}}^{\mathrm{eff}} & =\int_{0}^{R_{\mathrm{int}}} e^{-\beta U(r)} 4 \pi r^{2} \mathrm{~d} r,
\end{aligned}
$$

where $V_{\text {int }}$ is the interaction volume of the reactive particles, $V_{\text {int }}^{\text {eff }}$ the effective accessible volume due to particle interaction and $V_{\text {ex }}$ is the reduction of the accessible volume.

In phase $C$ the stationary distribution of positions $\mathbf{y}$ is

$$
p_{C}(\mathbf{y})=Z_{C}^{-1}
$$

with the partition function

$$
Z_{C}=\int \mathrm{d} \mathbf{y}=\iiint \mathrm{d} \mathbf{y}_{a} \mathrm{~d} \mathbf{y}_{b} \mathrm{~d} \mathbf{y}_{c}=V^{3}
$$

\section{Doi reaction model}

The microscopic reaction model is defined by the association rate function $\lambda^{+}(\mathbf{x})$ and the dissociation rate function $\lambda^{-}(\mathbf{y})$. The former describes the probability per unit time 
with which two particles $\mathrm{A}$ and $\mathrm{B}$ can react when the system is in phase $A B$ and depends on positions $\mathbf{x}$. The latter describes the probability per unit time with which a $\mathrm{C}$ particle dissociates into $\mathrm{A}$ and $\mathrm{B}$ when the system is in phase $C$. We assume that $\lambda^{+}(\mathbf{x})$ is radially symmetric, i.e. it only depends on $r=\left|\mathbf{x}_{b}-\mathbf{x}_{a}\right|$. Any microscopic reaction model, described by $\lambda^{+}(\mathbf{x})$ will result in an effective association rate $K_{\text {on }}^{\text {micro }}$ which reads

$$
K_{\mathrm{on}}^{\mathrm{micro}}=\int \lambda^{+}(\mathbf{x}) p_{A B}(\mathbf{x}) \mathrm{d} \mathbf{x}
$$

For $\lambda^{+}(\mathbf{x})$ and $\lambda^{-}(\mathbf{y})$ we use the Doi reaction model as depicted in Fig. 1, i.e. the microscopic association reaction rate function is a constant $\lambda_{\text {on }}$, when particles $\mathrm{A}$ and $\mathrm{B}$ are closer than the reaction radius $R_{\text {reac }}$

$$
\lambda^{+}(\mathbf{x})=\lambda_{\text {on }} \chi_{\text {reac }}(r) \text { with } \quad r=\left|\mathbf{x}_{b}-\mathbf{x}_{a}\right|,
$$

where $\chi_{\text {reac }}(r)$ indicates that $\mathrm{A}$ and $\mathrm{B}$ are within reactive distance

$$
\chi_{\text {reac }}(r)= \begin{cases}1, & \text { if } r<R_{\text {reac }} \\ 0, & \text { otherwise. }\end{cases}
$$

The microscopic dissociation rate function is constant and chosen equal to the macroscopic dissociation rate constant

$$
\lambda^{-}(\mathbf{y})=k_{\mathrm{off}} .
$$

We evaluate the effective microscopic association rate (11) for the Doi reaction model (12) and obtain

$$
\begin{aligned}
K_{\text {on }}^{\text {micro }} & =\lambda_{\text {on }} Z_{A B}^{-1} V^{2} \int_{0}^{R_{\text {reac }}} e^{-\beta U(r)} 4 \pi r^{2} \mathrm{~d} r \\
& =\lambda_{\text {on }} \frac{V_{\text {reac }}^{\text {eff }}}{V-V_{\text {ex }}}
\end{aligned}
$$

where the effective reaction volume $V_{\text {reac }}^{\text {eff }}$ takes a similar form as the effective interaction volume, but with another radius $R_{\text {reac }}$

$$
V_{\text {reac }}^{\text {eff }}=\int_{0}^{R_{\text {reac }}} e^{-\beta U(r)} 4 \pi r^{2} \mathrm{~d} r
$$

\section{Computing the microscopic association rate constant that reproduces the macroscopic equilibrium}

For the following we will assume a given dissociation constant $K_{d}$ and a given dissociation rate constant $k_{\text {off }}$. Using Eqs. $(3,2)$ we state the effective association rate according to the law of mass action

$$
K_{\mathrm{on}}=\frac{k_{\mathrm{off}}}{K_{d} V}
$$

We require that the micro- and macroscopic effective rates match

$$
K_{\text {on }}^{\text {micro }} \stackrel{!}{=} K_{\text {on }}
$$


and find the restrictions on the microscopic reaction model. This results in a choice for the microscopic association rate constant $\lambda_{\text {on }}$, that will yield the desired equilibrium as in Eq. (5). We will call this specific value $\tilde{\lambda}_{\text {on }}$

$$
\tilde{\lambda}_{\text {on }}=\frac{k_{\text {off }}}{K_{d} V} \frac{V-V_{\text {ex }}}{V_{\text {reac }}^{\text {eff }}}
$$

The relation of this expression to other diffusion influenced rate calculations is discussed in Appendix B.

\section{INTERACTING-PARTICLE REACTION DYNAMICS WITH DETAILED BALANCE}

Transition rates $k^{+}$and $k^{-}$of association $(+)$and dissociation $(-)$respectively between states $x_{A B}$ and $y_{C}$, with stationary probability distributions $p$ defined in Eq. (6) shall obey detailed balance

$$
p\left(x_{A B}\right) k^{+}(\mathbf{y} \mid \mathbf{x})=p\left(y_{C}\right) k^{-}(\mathbf{x} \mid \mathbf{y}) .
$$

We split the transition rates $k$ into proposal rate and acceptance probability

$$
\begin{aligned}
& k^{+}(\mathbf{y} \mid \mathbf{x})=\lambda^{+}(\mathbf{x}) q^{+}(\mathbf{y} \mid \mathbf{x}) \alpha^{+}(\mathbf{y} \mid \mathbf{x}) \text { association } \\
& k^{-}(\mathbf{x} \mid \mathbf{y})=\underbrace{\lambda^{-}(\mathbf{y}) q^{-}(\mathbf{x} \mid \mathbf{y})}_{\text {proposal }} \underbrace{\alpha^{-}(\mathbf{x} \mid \mathbf{y})}_{\text {acceptance }} \text { dissociation }
\end{aligned}
$$

where $\lambda^{+}(\mathbf{x})$ is the absolute rate of proposing a transition $\mathrm{A}+\mathrm{B} \rightarrow \mathrm{C}$ when in particle configuration $\mathbf{x} \cdot q^{+}(\mathbf{y} \mid \mathbf{x})$ is the normalized density to propose the positions $\mathbf{y}$, given that the positions were $\mathbf{x} \cdot \alpha^{+}(\mathbf{y} \mid \mathbf{x})$ is the absolute probability of accepting the proposed positions. Similarly $\lambda^{-}(\mathbf{y})$ is the absolute rate of proposing a transition $\mathrm{C} \rightarrow \mathrm{A}+\mathrm{B}, q^{-}(\mathbf{x} \mid \mathbf{y})$ is the according proposal density and $\alpha^{-}(\mathbf{x} \mid \mathbf{y})$ the absolute probability of accepting the proposal. All $q$ and $\alpha$ satisfy

$$
\int q^{i}(\mathbf{y} \mid \mathbf{x}) \mathrm{d} \mathbf{y}=1 \quad \text { and } \quad \alpha^{i}(\mathbf{y} \mid \mathbf{x}) \leq 1 \quad \text { for } i \in\{+,-\}
$$

\section{A. Derive the backward proposal from the forward proposal}

We assume the association proposal density $q^{+}$as given, and want to derive the dissociation proposal density $q^{-}$and both $\alpha^{+}$and $\alpha^{-}$subject to detailed balance. Therefore we include all terms that depend on the particle positions into the reverse proposal density $q^{-}$, such that most terms in Eq. (20) cancel and acceptances $\alpha^{+}$and $\alpha^{-}$become independent of the particle positions of the dissociated phase. The reverse proposal density reads

$$
q^{-}(\mathbf{x} \mid \mathbf{y})=Q(\mathbf{y})^{-1} q^{+}(\mathbf{y} \mid \mathbf{x}) \frac{\lambda^{+}(\mathbf{x})}{\lambda^{-}(\mathbf{y})} \frac{p_{A B}(\mathbf{x})}{p_{C}(\mathbf{y})}
$$

with the normalization function $Q(\mathbf{y})$ such that

$$
Q(\mathbf{y})=\frac{1}{\lambda^{-}(\mathbf{y}) p_{C}(\mathbf{y})} \int q^{+}(\mathbf{y} \mid \mathbf{x}) \lambda^{+}(\mathbf{x}) p_{A B}(\mathbf{x}) \mathrm{d} \mathbf{x}
$$


Note that $Q$ must depend on $\mathbf{y}$ to fulfil the normalization $\forall \mathbf{y}$ (in the Doi model it will reduce to a constant). Inserting Eqs. $(22,21)$ into Eq. (20), the detailed balance condition reduces to

$$
\frac{\alpha^{+}(\mathbf{y} \mid \mathbf{x})}{\alpha^{-}(\mathbf{x} \mid \mathbf{y})}=\frac{\pi_{C}}{\pi_{A B}} \frac{1}{Q(\mathbf{y})}
$$

Reminding that $\alpha \leq 1$ naturally leads to using the Metropolis-Hastings $[50,51]$ acceptance function

$$
\begin{aligned}
& \alpha^{+}(\mathbf{y} \mid \mathbf{x})=\min \left\{1, \frac{\pi_{C}}{\pi_{A B} Q(\mathbf{y})}\right\} \\
& \alpha^{-}(\mathbf{x} \mid \mathbf{y})=\min \left\{1, \frac{\pi_{A B} Q(\mathbf{y})}{\pi_{C}}\right\}
\end{aligned}
$$

which fulfils the given detailed balance condition (24). For a practical implementation one needs to know both proposal densities $q^{+}(\mathbf{y} \mid \mathbf{x})$ and $q^{-}(\mathbf{x} \mid \mathbf{y})$, and the corresponding acceptance probabilities $\alpha^{+}(\mathbf{y} \mid \mathbf{x})$ and $\alpha^{-}(\mathbf{x} \mid \mathbf{y})$.

\section{B. Apply DB to Doi model}

Assuming the Doi model $(12,14)$, we state the association proposal density $q^{+}(\mathbf{y} \mid \mathbf{x})$ and derive the dissociation proposal density $q^{-}(\mathbf{x} \mid \mathbf{y})$ (22). The normalized association proposal density reads

$$
q^{+}(\mathbf{y} \mid \mathbf{x})=V^{-2} \delta\left(\mathbf{y}_{c}-\frac{\mathbf{x}_{a}+\mathbf{x}_{b}}{2}\right)
$$

where the Dirac delta function $\delta(\cdot)$ assures that the $\mathrm{C}$ particle'sproposed position $\mathbf{y}_{c}$ is in the middle between the $\mathrm{A}$ and $\mathrm{B}$ particles from the initial positions $\mathbf{x}$. The volume term $V^{-2}$ is required for normalization, due to the measure $\mathrm{d} \mathbf{y}=\mathrm{d} \mathbf{y}_{a} \mathrm{~d} \mathbf{y}_{b} \mathrm{~d} \mathbf{y}_{c}$. Additionally the volume term can be understood as a uniform placement of $\mathrm{A}$ and $\mathrm{B}$ in the final positions y. Since A and B are not considered in the associated state, it is irrelevant where they are. Hence Eq. (26) fulfils $\int q^{+}(\mathbf{y} \mid \mathbf{x}) \mathrm{d} \mathbf{y}=1$. The normalization $Q$ of the dissociation proposal density from Eq. (23) can be evaluated and reduces to a constant (see Appendix C)

$$
Q=\frac{\lambda_{\mathrm{on}}}{k_{\mathrm{off}}} \frac{V_{\mathrm{reac}}^{\mathrm{eff}}}{V-V_{\mathrm{ex}}} .
$$

The dissociation proposal density (22) then becomes

$$
\begin{aligned}
q^{-}(\mathbf{x} \mid \mathbf{y})= & \left(V V_{\text {reac }}^{\mathrm{eff}}\right)^{-1} \delta\left(\mathbf{y}_{c}-\frac{\mathbf{x}_{a}+\mathbf{x}_{b}}{2}\right) \ldots \\
& \times \chi_{\text {reac }}(r) e^{-\beta U(r)}
\end{aligned}
$$

with $r=\left|\mathbf{x}_{b}-\mathbf{x}_{a}\right|$. This density can be read as: given a $\mathrm{C}$ particle at position $\mathbf{y}_{c}$, positions $\mathbf{x}_{a}$ and $\mathbf{x}_{b}$ of particles $\mathrm{A}$ and $\mathrm{B}$ are restricted to radial shells concentric around $\mathbf{y}_{c}$ due to the delta function. These shells must not be larger than the reaction radius due to the indicator function. The distance is additionally weighted with the Boltzmann factor of the interaction potential $U$.

Using the normalization constant $Q$ from Eq. (27) the acceptance probabilities from Eq. (25) are directly obtained. Using the microscopic association rate given in Eq. (19) results in an acceptance probability of unity in both directions

$$
\alpha^{+}(\mathbf{y} \mid \mathbf{x})=\alpha^{-}(\mathbf{x} \mid \mathbf{y})=1 \quad \text { for } \lambda_{\text {on }}=\tilde{\lambda}_{\text {on }} \text { from (19). }
$$




\section{Generalize for other types of reactions}

The presented Metropolis-Hastings Monte Carlo method can be performed for other types of reversible reactions, namely reversible conversion reactions of the type

$$
\underbrace{\mathrm{A}}_{\mathbf{x}} \underbrace{\stackrel{k_{\text {on }}}{\rightleftarrows}}_{k_{\text {off }}} \underbrace{\mathrm{B}}_{\mathbf{y}} \text { with } \lambda^{+}(\mathbf{x}), \lambda^{-}(\mathbf{y})
$$

as well as reversible enzymatic reactions of the type

$$
\underbrace{\mathrm{A}+\mathrm{C}}_{\mathbf{x}} \underbrace{\stackrel{k_{\mathrm{on}}}{\rightleftarrows}}_{k_{\mathrm{off}}} \underbrace{\mathrm{B}+\mathrm{C}}_{\mathbf{y}} \quad \text { with } \lambda^{+}(\mathbf{x}), \lambda^{-}(\mathbf{y}) \text { and } R_{\mathrm{reac}}
$$

with macroscopic forward and backward rates $k_{\text {on }}$ and $k_{\text {off }}$. For those two reactions we can also construct a microscopic probability density for positions $\mathbf{x}$ and $\mathbf{y}$ for the dilute case in the fashion of Eq. (6). Here the microscopic phase space only has positions for A and B particles, the $\mathrm{C}$ particle in reaction (30) can be placed at the origin without loss of generality. The reaction functions $\lambda^{+}(\mathbf{x})$ and $\lambda^{-}(\mathbf{y})$ for the conversion reaction (29) are constants $\lambda_{\text {on }}$ and $\lambda_{\text {off }}$ respectively. For the enzymatic reaction (30) both reaction functions are additionally multiplied with an indicator function depending on the reaction radius $R_{\text {reac }}$. As in Sec. IID we can compute the microscopic rate constants $\lambda$ that reproduce the macroscopic kinetics in the dilute limit. In the case of the enzymatic reaction (30), there appear excluded volumes $V_{\text {ex,A }}, V_{\text {ex,B }}$ and effective reaction volumes $V_{\text {reac,A }}^{\text {eff }}, V_{\text {reac,B }}^{\text {eff }}$. These are defined analogously to the volumes $V_{\text {ex }}$, see Eq. (8), and $V_{\text {reac }}^{\text {eff }}$, see Eq. (16), with the difference that $V_{\text {ex,A }}$ and $V_{\text {reac, }}^{\text {eff }}$ are calculated based on the interaction potential of $\mathrm{A}$ and $\mathrm{C}$, and $V_{\text {ex,B }}$ and $V_{\text {reac,B }}^{\text {eff }}$ are calculated based on the interaction potential of B and C. To assure detailed balance we make the same ansatz for transition rates as in Eq. (21). The proposal densities $q$ are constructed much simpler, because in these types of reactions no new positions must be generated, i.e. the $q$ are delta functions. However during the species conversion, molecules might be subject to potentials with respect to educt and product states. We gather the change of potential energy during the reaction in the variable $\Delta E$. We summarize all of these findings in Tab. III.

\section{RESULTS}

We have proposed a method of executing reversible reactions according to detailed balance. It can be used to perform reactions in a stochastic reaction-diffusion simulation. A schematic implementation is shown in the pseudo code Alg. 1.

In order to illustrate our method, we perform many-particle simulations with molecular species $\mathrm{A}, \mathrm{B}$ and $\mathrm{C}$ engaging in the reversible association reaction shown in Eq. (1). The simulation is performed using overdamped Langevin dynamics in the particle interaction potential with a fixed time-step integrator. The potential $U(r)$ between the particles A and $\mathrm{B}$ is chosen as a harmonic repulsion with cutoff $R_{\text {int }}$ and force constant $\kappa$, that only depends on the distance $r=\left|\mathbf{x}_{b}-\mathbf{x}_{a}\right|$ between $\mathrm{A}$ and $\mathrm{B}$

$$
U(r)=\left\{\begin{aligned}
\frac{1}{2} \kappa\left(r-R_{\mathrm{int}}\right)^{2}, & \text { if } r<R_{\mathrm{int}} \\
0, & \text { otherwise }
\end{aligned}\right.
$$


For this choice of potential the effective interaction volume from Eq. (10) yields an expression containing errorfunctions. In general the effective interaction volume can be determined numerically.

During one time step of length $\tau$, we first integrate the diffusive motion of particles and then perform the reactions. The boundaries of the system are periodic, obeying the minimum image convention and wrapping positions upon crossing the border.

In the reaction step all possible reaction events are determined, this depends on the considered reactions, reaction radii and the current particle configuration. Then the list of reaction events is processed. An event is selected from the list. The event will be proposed with absolute probability $p=1-\exp (-\lambda \tau)$ depending on the microscopic rate constant $\lambda$ of the associated type of reaction. The event is performed, generating another particle configuration drawn from the proposal densities in Eqs. $(26,28)$. From the change in potential energy and the type of reaction the acceptance probability $a$ is calculated. If the event is accepted the new configuration is kept. If the event is rejected the old configuration has to be restored. Then the processed event is removed from the list of events. Additionally any event is removed that would propose an event with the same particles as the processed one, since these might not exist anymore.

The total probability of performing a particular event is $p a$. If $a$ is chosen according to Eq. (25) and Eq. (27) and the proposal density of the dissociation reaction includes the Boltzmann factor as in Eq. (28), we will refer to this as the proposed DB reaction scheme. We refer to the Doi reaction scheme if $a=1$, regardless of the energy difference, and if the proposal density does not include the Boltzmann factor of the interaction potential of the reactants.

\section{A. Dilute limit}

We validate Alg. 1 by performing it on the system of particles A, B and C. These particles are subject to the reaction (1) and a harmonic repulsion potential as in Eq. (31). At any point in time there is either the $\mathrm{C}$ particle or two particles $\mathrm{A}$ and $\mathrm{B}$, i.e. there is only one instance of each molecule species. Thus these simulations are in the dilute limit. The only interactions occur between the A and B particle.

\section{Validation of reaction kinetics}

We show that the proposed detailed balance reaction scheme always yields the desired macroscopic equilibrium distribution $\pi_{A B} / \pi_{C}$ from Eq. (5). Additionally we demonstrate under which circumstances the simulated effective on- and off-rates, $K_{\text {on }}$ and $K_{\text {off }}$, will match those given by Eq. (3) and Eq. (4). The results are seen in Fig. 2. The simulation parameters are given in Tab. I.

Fig. 2a shows that for very low $\lambda_{\text {on }}$, the effective association rate $K_{\text {on }}$ cannot exceed a certain value because the proposal frequency is limited and $K_{\text {off }}$ is in turn diminished by rejection of dissociation events in order to reproduce the desired equilibrium constant $\pi_{A B} / \pi_{C}=K_{d} V$. For very high $\lambda_{\text {on }}$, association events will be rejected, thus limiting $K_{\text {on }}$ to the LMA value, while dissociation events are executed with frequency $K_{\text {off }}=k_{\text {off }}$. The transition between these two regimes is where $\lambda_{\text {on }}=\tilde{\lambda}_{\text {on }}$ as in Eq. (19). Fig. 2b shows that, when one uses the appropriate association rate constant from Eq. (19), one can reproduce the expected reaction kinetics for varying $K_{d}$. 


\section{Microscopic reversibility}

We now demonstrate that the proposed DB reaction scheme (Alg. 1) indeed produces trajectories in thermodynamic equilibrium, while the naive Doi scheme leads to periodic cycles in phase space, corresponding to an unintended nonequilibrium scenario. To this end, we distinguish three substates of the dissociated state, defined by the inter-particle distance $r$ of particles $\mathrm{A}$ and $\mathrm{B}$, and the reaction radius $R$. We define states $1-4$ as follows:

1. The complex state, $\mathrm{C}$

2. A and B are very close $r \leq \frac{3}{4} R$

3. A and $\mathrm{B}$ are still in reactive range $\frac{3}{4} R<r \leq R$

4. $\mathrm{A}$ and $\mathrm{B}$ are not within reactive range $r>R$

Using again a reversibly reacting system with a single A, B pair or a single C complex, we determine the stationary distribution $\pi$ for this definition of states, and the transition rates $K$ connecting them. A process that fulfils detailed balance must yield

$$
\pi_{i} K_{i j}=\pi_{j} K_{j i}
$$

for all pairs of states $i, j$. We measure $\pi$ and $K$ from simulations and compare the Doi reaction scheme and the proposed $\mathrm{DB}$ reaction scheme in the presence of a harmonic repulsion potential between $\mathrm{A}$ and $\mathrm{B}$. In this comparison all system parameters are identical, only the reaction mechanism differs. Results are presented in Fig. 3 and simulation parameters are given in Tab. I.

From Fig. 3 it is evident that for the present case of interacting particles, the naive Doi reaction scheme produces a cyclic probability flux that violates DB. In the proposed DB reaction scheme, this is not the case and all given probability fluxes obey Eq. (32).

Note that for both reaction schemes, there occurs a unidirectional transition $4 \rightarrow 1$ due to the time splitting we employ during one simulation step (first the diffusion step and then the reaction step). This artificial transition is a result of the time-step discretization error and not related to the DB scheme. It occurs with an absolute rate of less than $10^{-6}$, all other transitions have $K_{i j}>10^{-5} \forall(i, j) \neq(4,1)$. Thus its probability flux is not shown here.

\section{B. System of many particles}

Finally, we study how a dense mixture of interacting particles behaves when the DB algorithm is employed, and we compare this behavior with the naive Doi algorithm and what is expected from physical intuition. The Algorithm 1 is performed for a system of many A, B and $\mathrm{C}$ particles confined to the volume $V$ with periodic boundaries. In this scenario we assign physical radii $r_{A}, r_{B}$, and $r_{C}$ to the particles. Particles are subject to harmonic repulsion potentials (31) acting between all pairs of species A, B, and C, where the interaction radius is chosen as the sum of the particles' radii. See parameters in Tab. II. Particles are subject to the reaction (1). Employing the DB reaction scheme introduced in Sec. III can therefore result in rejected Monte-Carlo moves, which will affect the thermodynamics and kinetics of the simulation system in the dense limit.

In Sec. II B and following we had assumed that phase space consists of only three particles $\mathrm{A}, \mathrm{B}$ and $\mathrm{C}$. In the case of many possible reactants one is presented with multiple possible 
reaction events. For one particular event we will use the proposal densities from Eqs. $(26,28)$ to treat the particles taking part in the event. All other particles will be considered static excess objects. This means that the microscopic distributions from Eq. (6) gain another Boltzmann factor from interactions with the excess particles. Note that the partition functions $Z_{A B}$ and $Z_{C}$ will differ from their "dilute" values. In Sec. III B we have seen that a particular choice of parameters leads to the prefactor in the acceptance becoming unity. Hence, the advantage of such a Markov Chain Monte Carlo algorithm is that one does not need to know constant factors of the stationary distribution to draw samples from said distribution. Along these lines we construct an acceptance function for the many particle case, that includes a Boltzmann factor of the energy difference and a prefactor of unity, assuming that internal reaction parameters correspond to a certain but unknown macroscopic equilibrium. We will use the association rate constant derived in Eq. (19). Obviously this equilibrium will differ from the one in Eq. (5). But one can guarantee detailed balance never the less.

The change of potential energy is $\Delta \epsilon$. It does not include the interaction between $\mathrm{A}$ and $\mathrm{B}$ as this is already accounted for by the proposal probabilities $q^{+}$and $q^{-}$. We may write $\Delta \epsilon$ as the total change of potential energy $\Delta E$ minus the interaction energy $U_{A B}$. We formulate the acceptance for the many particle case:

$$
\begin{aligned}
& \alpha^{+}(\mathbf{y} \mid \mathbf{x})=\min \left\{1, \exp \left(-\beta \Delta \epsilon^{+}\right)\right\} \\
& \alpha^{-}(\mathbf{x} \mid \mathbf{y})=\min \left\{1, \exp \left(-\beta \Delta \epsilon^{-}\right)\right\}
\end{aligned}
$$

where the changes of energies are given by

$$
\begin{aligned}
& \Delta \epsilon^{+}=E(\mathbf{y})-\left[E(\mathbf{x})-U_{A B}(\mathbf{x})\right] \\
& \Delta \epsilon^{-}=\left[E(\mathbf{x})-U_{A B}(\mathbf{x})\right]-E(\mathbf{y}) .
\end{aligned}
$$

We set up the system with a certain number of $\mathrm{A}$ and $\mathrm{B}$ particles and no $\mathrm{C}$ particles. We control the quantity $n=\left(N_{A}+N_{B}\right) / 2+N_{C}$ which is conserved during a simulation. The system equilibrates without the reaction, we then switch the reaction on and let the system equilibrate again.

We compute three observables in the equilibrated state, i.e. when observables are stable and converged: the equilibrium constant $\pi_{A B} / \pi_{C}=V[A][B] /[C]$, the total potential energy of the system $U$ in units of $k_{B} T$ and the pressure $P$ in units of $V^{-1} k_{B} T$. The pressure is measured from evaluating the virial term of acting forces as described in [53]. Individual reactions are integrated with either the proposed DB scheme or the Doi reaction scheme.

Fig. 4a shows the results for the case when an association reaction of $\mathrm{A}$ and $\mathrm{B}$ increases the total volume occupied by particles such that $r_{A}^{3}+r_{B}^{3}<r_{C}^{3}$. The associated state is energetically less favourable. In the dilute limit both methods Doi and DB reproduce the macroscopic equilibrium population $\pi_{A B} / \pi_{C}=K_{d} V$. For increasing number of particles both methods differ significantly. The Doi reaction scheme favours the energetically higher associated configuration $\mathrm{C}$. The Doi scheme produces an equilibrium constant of roughly $\pi_{A B} / \pi_{C} \approx 80$ for the highest density simulated. The DB scheme adjusts the effective association probability by rejecting association events. This results in a steady state, where almost no $C$ particles exist with an equilibrium constant exceeding $\pi_{A B} / \pi_{C}>3 \times 10^{3}$. For all $n>50$, the DB scheme finds a steady state of lower energy and lower pressure compared to the Doi scheme. Fig. 5a and b show representative simulation snapshots of the steady states for Doi and DB scheme.

Fig. 4b shows the case when a $\mathrm{C}$ particle occupies less volume than $\mathrm{A}$ and $\mathrm{B}$ combined such that $r_{A}^{3}+r_{B}^{3}>r_{C}^{3}$, which could correspond to two proteins $\mathrm{A}$ and $\mathrm{B}$, which only fully fold in 
a bound state. In the dilute case both methods Doi and DB reproduce the same behaviour in all three observables. For increasing number of particles the Doi method produces a similar steady state population as in Fig. 4a where the $C$ state is favoured. The DB scheme produces states favouring the $C$ state even stronger thus reducing the system's potential energy and pressure compared to the Doi scheme. Fig. $5 \mathrm{c}$ and $\mathrm{d}$ show representative simulation snapshots of the steady states for Doi and DB scheme.

\section{CONCLUSION}

We have derived an algorithm to perform iPRD simulations of molecules undergoing reversible reactions of the form $\mathrm{A}+\mathrm{B} \rightleftarrows \mathrm{C}$ according to detailed balance. This method is called iPRD-DB.

Detailed balance guarantees that simulations of an isolated system generate samples according to thermodynamic equilibrium. We have shown that in a dense reactive mixture of particles, that exhibit volume exclusion due to pair-wise potentials, the steady state of the system simulated with iPRD-DB is in agreement with Henri Le Chatelier's principle [52], i.e. that the achieved steady state concentrations strongly depend on the interaction of molecules. Biochemical pathways often show switch-like behavior, and are thus sensitive to such changes in concentrations of agents [54-56]. Sampling the correct equilibrium is crucial when simulating such processes.

The iPRD-DB method can be generalized for other types of reactions, such as a reversible change of molecule species $\mathrm{A} \rightleftarrows \mathrm{B}$, or a reversible enzymatic reaction $\mathrm{A}+\mathrm{C} \rightleftarrows \mathrm{B}+\mathrm{C}$, which describes a Michaelis-Menten experiment when the backwards rate becomes very small.

Furthermore the iPRD-DB method is accompanied by an equation for the microsopic rate constant $\lambda$ that assures the correct macroscopic reaction kinetics. This equation, see Eq. (19), relates the macroscopic kinetic parameters $K_{d}$ and $k_{\text {off }}$ in a dilute environment with the microscopic iPRD model parameters: microscopic rate constant $\lambda$, reaction radius $R$, and force parameters that determine the excluded volume $V_{\text {ex }}$. Thus, it provides a choice for $\lambda$, which in the iPRD-DB algorithm functions as the absolute proposal rate. For this choice the acceptance probability reduces to the Boltzmann factor describing the change of energy with respect to educt and product states. We also provide proposal densities such that the acceptance becomes unity in the dilute case.

Having measured $K_{d}$ and $k_{\text {off }}$ in an in vitro scenario, a microscopic iPRD model can be constructed subject to Eq. (19) and can then be analyzed numerically to gain insights about the in vivo process, where molecules may occur in very low copy numbers and diffuse anomalously due to complex geometries, making experimental measurements cumbersome in this regime. Note that the expression relating $K_{d}$ and $k_{\text {off }}$ with $\lambda$ and $R$ is independent of the diffusion coefficient $D$, i.e. an iPRD model can be adjusted to resemble the in vivo effective diffusion, which may, e.g. be obtained from Förster resonance energy transfer (FRET) experiments [57].

An open question is what the analytical reference chemical equilibrium is when going to dense particle mixtures.

\section{ACKNOWLEDGEMENTS}

This paper is dedicated to William A. Eaton on the occasion of his 80th birthday. Happy Birthday Bill - you are a wonderful person and your work has been an inspiration for this field! 
We gratefully acknowledge funding from Deutsche Forschungsgemeinschaft (SFB 958 / Project A04, TRR 186 / Project A12, SFB 1114 / Project C03), Einstein Foundation Berlin (ECMath Project CH17) and European Research Council (ERC CoG 772230 "ScaleCell"). We are grateful for inspiring discussions with Moritz Hoffmann, Manuel Dibak, Luigi Sbailò, Mohsen Sadeghi, Felix Höfling and Christof Schütte.

\section{APPENDIX}

\section{A. Normalization constant $Z_{A B}$}

The normalization is

$$
\begin{aligned}
Z_{A B} & =\int e^{-\beta U(\mathbf{x})} \mathrm{d} \mathbf{x} \\
& =\int \mathrm{d} \mathbf{x}_{c} \iint e^{-\beta U\left(\mathbf{x}_{b}-\mathbf{x}_{a}\right)} \mathrm{d} \mathbf{x}_{a} \mathrm{~d} \mathbf{x}_{b} \\
& =V\left(I_{1}+I_{2}\right)
\end{aligned}
$$

If there are no external potentials present, the latter integral factorizes

$$
\begin{aligned}
I_{2} & =\iint_{\left|\mathbf{x}_{b}-\mathbf{x}_{a}\right|>R_{\mathrm{int}}} \mathrm{d} \mathbf{x}_{a} \mathrm{~d} \mathbf{x}_{b} \\
& =\int\left(\int_{\left|\mathbf{x}_{b}-\mathbf{x}_{a}\right|>R_{\text {int }}} \mathrm{d} \mathbf{x}_{b}\right) \mathrm{d} \mathbf{x}_{a} \\
& =\left(V-V_{\text {int }}\right) \int \mathrm{d} \mathbf{x}_{a}=\left(V-V_{\text {int }}\right) V
\end{aligned}
$$

where $V_{\text {int }}$ is the interaction volume, that only depends on the cut-off distance of the potential $R_{\text {in }}$, not the potential itself. Since the potential $U$ only depends on the relative position $\mathbf{x}_{b}-\mathbf{x}_{a}$, one can fix the position of one particle without changing the value of the integral $I_{1}$

$$
\begin{aligned}
I_{1} & =\iint_{\left|\mathbf{x}_{b}-\mathbf{x}_{a}\right| \leq R_{\mathrm{int}}} e^{-\beta U\left(\mathbf{x}_{b}-\mathbf{x}_{a}\right)} \mathrm{d} \mathbf{x}_{a} \mathrm{~d} \mathbf{x}_{b} \\
& =\int\left(\int_{\left|\mathbf{x}_{b}-\mathbf{x}_{a}\right| \leq R_{\mathrm{int}}} e^{-\beta U\left(\mathbf{x}_{b}-\mathbf{x}_{a}\right)} \mathrm{d} \mathbf{x}_{b}\right) \mathrm{d} \mathbf{x}_{a} \\
& =V_{\text {int }}^{\text {eff }} \int \mathrm{d} \mathbf{x}_{a}=V_{\text {int }}^{\text {eff }} V
\end{aligned}
$$

The effective accessible volume inside the interaction radius is given by:

$$
V_{\text {int }}^{\mathrm{eff}}=V_{\mathrm{int}}-V_{\mathrm{ex}},
$$

which defines the excluded volume $V_{\text {ex }}$ due to interaction

\section{B. Relation to diffusion-influenced rate constant derivations}

To understand Eq. (19) we formulate the association rate constant for our problem using Eq. (2)

$$
k_{\mathrm{on}}=\tilde{\lambda}_{\mathrm{on}} V \frac{V_{\mathrm{reac}}^{\mathrm{eff}}}{V-V_{\mathrm{ex}}} .
$$


This rate is linearly dependent on the effective reaction volume from Eq. (16), i.e. if one increases the repulsion force between particles A and B the association rate will decrease. One further notices that the diffusion of particles is not considered in this equation, since we assume they are at all times distributed according to Eq. (6). This is true only because of the reversible reaction that the isolated pair is subject to. The diffusion approach of $\mathrm{A}$ and $\mathrm{B}$ need not be considered here. It is therefore crucial in an algorithm to generate samples from the stationary distribution we assumed.

At this point we can establish a connection with other treatments of diffusion influenced reaction rates. The formula derived by Doi [44] describes the association rate constant for particles approaching each other via diffusion from the far-field. It includes the relative diffusion constant of the two particles $D$ and reads

$$
k_{\text {on,Doi }}=4 \pi D R\left(1-\sqrt{\frac{D}{\lambda_{\text {on }} R^{2}}} \tanh \left(\sqrt{\frac{\lambda_{\text {on }} R^{2}}{D}}\right)\right)
$$

Assuming the fast diffusion limit of this yields [1]

$$
\lambda \ll \frac{D}{R^{2}} \rightarrow k_{\mathrm{on}, \text { Doi }} \approx \lambda_{\mathrm{on}} \frac{4}{3} \pi R^{3}
$$

If we on the other hand assume the large volume limit of the expression from Eq. (35) we arrive at

$$
R^{3} \ll V \quad \rightarrow \quad k_{\text {on }}=\tilde{\lambda}_{\text {on }} V_{\text {reac }}^{\text {eff }} .
$$

Comparing Eqs. $(36,37)$ we see that they match if the term $4 \pi R^{3} / 3$ is identified as the effective reaction volume without potentials.

\section{Normalization of dissociation proposal density}

Additionally we need $Q(\mathbf{y})$ from 23

$$
\begin{aligned}
Q(\mathbf{y})= & \frac{\lambda_{\mathrm{on}} V}{k_{\mathrm{off}} Z_{A B}} \iiint \delta\left(\mathbf{y}_{c}-\frac{\mathbf{x}_{a}+\mathbf{x}_{b}}{2}\right) \chi_{\mathrm{reac}}(\mathbf{x}) \ldots \\
& \times e^{-\beta U\left(\left|\mathbf{x}_{b}-\mathbf{x}_{a}\right|\right)} \mathrm{d} \mathbf{x}_{a} \mathrm{~d} \mathbf{x}_{b} \mathrm{~d} \mathbf{x}_{c} \\
= & \frac{\lambda_{\mathrm{on}} V^{2}}{k_{\mathrm{off}} Z_{A B}} \iint_{\left|\mathbf{x}_{b}-\mathbf{x}_{a}\right| \leq R} \delta\left(\mathbf{y}_{c}-\frac{\mathbf{x}_{a}+\mathbf{x}_{b}}{2}\right) \ldots \\
& \times e^{-\beta U\left(\left|\mathbf{x}_{b}-\mathbf{x}_{a}\right|\right)} \mathrm{d} \mathbf{x}_{a} \mathrm{~d} \mathbf{x}_{b}
\end{aligned}
$$

The delta function can be reformulated in relative coordinates of $\mathrm{A}$ and $\mathrm{B}$, that have to placed symmetric around $\mathbf{y}_{c}$. This eliminates another integral, which yields 1 , due to the delta function. The only remaining degree of freedom is the distance of $\mathrm{A}$ and $\mathrm{B}$, which results in an integral, that is identical to the effective reaction volume $V_{\text {reac }}^{\text {eff }}$ from Eq. (16).

[1] R. Erban, J. Chapman, and S. J. Chapman, "Stochastic modelling of reaction-diffusion processes: algorithms for bimolecular reactions.," Physical biology, vol. 6, no. 4, p. 046001, 2009-01. 
[2] J. S. van Zon and P. R. ten Wolde, "Green's-function reaction dynamics: a particle-based approach for simulating biochemical networks in time and space.," The Journal of chemical physics, vol. 123, no. 2005, p. 234910, 2005.

[3] M. Hoffmann and U. S. Schwarz, "Oscillations of Min-proteins in micropatterned environments: a three-dimensional particle-based stochastic simulation approach.," Soft matter, vol. 10, no. 14, pp. 2388-96, 2014.

[4] S. S. Andrews, "Smoldyn: Particle-based simulation with rule-based modeling, improved molecular interaction and a library interface," Bioinformatics, vol. 33, no. 5, pp. 710-717, 2017.

[5] S. R. Agarwal, C. E. Clancy, and R. D. Harvey, "Mechanisms Restricting Diffusion of Intracellular cAMP," Scientific Reports, vol. 6, no. 1, p. 19577, 2016-04.

[6] D. Albrecht, C. M. Winterflood, M. Sadeghi, T. Tschager, F. Noé, and H. Ewers, "Nanoscopic compartmentalization of membrane protein motion at the axon initial segment," J. Cell Biol., vol. 215 , pp. $37-46,2016$.

[7] M. B. Elowitz, "Stochastic Gene Expression in a Single Cell," Science, vol. 297, no. 5584, pp. 1183-1186, 2002-08.

[8] U. S. Bhalla, "Signaling in Small Subcellular Volumes. I. Stochastic and Diffusion Effects on Individual Pathways," Biophysical Journal, vol. 87, no. 2, pp. 733-744, 2004-08.

[9] G. J. Doherty and H. T. McMahon, "Mechanisms of Endocytosis," Annual Review of Biochemistry, vol. 78, no. 1, pp. 857-902, 2009-06.

[10] T. D. Pollard and G. G. Borisy, "Cellular motility driven by assembly and disassembly of actin filaments," Cell, vol. 112, no. 4, pp. 453-465, 2003.

[11] S. K. Sadiq, "Reaction-diffusion basis of retroviral infectivity," Philosophical Transactions of the Royal Society A: Mathematical, Physical and Engineering Sciences, vol. 374, no. 2080, p. 20160148, 2016-11.

[12] J. S. van Zon and P. R. Ten Wolde, "Simulating biochemical networks at the particle level and in time and space: Green's function reaction dynamics," Physical review letters, vol. 94, no. 12, p. $128103,2005$.

[13] K. Takahashi, S. Tanase-Nicola, and P. R. ten Wolde, "Spatio-temporal correlations can drastically change the response of a MAPK pathway," Proceedings of the National Academy of Sciences, vol. 107, no. 6, pp. 2473-2478, 2010-02.

[14] T. Opplestrup, V. V. Bulatov, G. H. Gilmer, M. H. Kalos, and B. Sadigh, "First-passage Monte Carlo algorithm: Diffusion without all the hops," Physical Review Letters, vol. 97, no. 23, pp. 10-13, 2006.

[15] A. Donev, V. V. Bulatov, T. Oppelstrup, G. H. Gilmer, B. Sadigh, and M. H. Kalos, "A First-Passage Kinetic Monte Carlo algorithm for complex diffusion-reaction systems," Journal of Computational Physics, vol. 229, no. 9, pp. 3214-3236, 2010.

[16] J. Schöneberg and F. Noé, "ReaDDy-a software for particle-based reaction-diffusion dynamics in crowded cellular environments.," PloS one, vol. 8, no. 9, p. e74261, 2013.

[17] J. Schöneberg, A. Ullrich, and F. Noé, "Simulation tools for particle-based reaction-diffusion dynamics in continuous space," BMC Biophysics, vol. 7, no. 1, p. 11, 2014-12.

[18] J. Biedermann, A. Ullrich, J. Schöneberg, and F. Noé, "ReaDDyMM: Fast interacting particle reaction-diffusion simulations using graphical processing units.," Biophysical journal, vol. 108, no. 3, pp. 457-61, 2015-02.

[19] F. Höfling and T. Franosch, "Anomalous transport in the crowded world of biological cells," arXiv, pp. 1-55, 2013.

[20] M. Gunkel, J. Schöneberg, W. Alkhaldi, S. Irsen, F. Noé, U. B. Kaupp, and A. Al-Amoudi, "Higher-Order Architecture of Rhodopsin in Intact Photoreceptors and Its Implication for 
Phototransduction Kinetics," Structure, vol. 23, no. 4, pp. 628-638, 2015-04.

[21] J. Schöneberg, M. Heck, K. P. Hofmann, and F. Noé, "Explicit Spatiotemporal Simulation of Receptor-G Protein Coupling in Rod Cell Disk Membranes," Biophysical Journal, vol. 107, no. 5, pp. 1042-1053, 2014.

[22] M. Hoffmann, C. Fröhner, and F. Noé, "ReaDDy 2." https://readdy.github.io.

[23] M. Sadeghi, T. R. Weikl, and F. Noé, "Particle-based membrane model for mesoscopic simulation of cellular dynamics," The Journal of Chemical Physics, vol. 148, no. 4, p. 044901, 2018-01.

[24] A. Ullrich, M. A. Böhme, J. Schöneberg, H. Depner, S. J. Sigrist, and F. Noé, "Dynamical Organization of Syntaxin-1A at the Presynaptic Active Zone," PLOS Computational Biology, vol. 11, no. 9, p. e1004407, 2015-09.

[25] Y. Posor, M. Eichhorn-Gruenig, D. Puchkov, J. Schöneberg, A. Ullrich, A. Lampe, R. Müller, S. Zarbakhsh, F. Gulluni, E. Hirsch, M. Krauss, C. Schultz, J. Schmoranzer, F. Noé, and V. Haucke, "Spatiotemporal control of endocytosis by phosphatidylinositol-3,4-bisphosphate," Nature, vol. 499, no. 7457, pp. 233-237, 2013-07.

[26] J. Schöneberg, M. Lehmann, A. Ullrich, Y. Posor, W.-t. T. Lo, G. Lichtner, J. Schmoranzer, V. Haucke, and F. Noé, "Lipid-mediated PX-BAR domain recruitment couples local membrane constriction to endocytic vesicle fission," Nature Communications, vol. 8, no. May, p. 15873, 2017.

[27] A. Vijaykumar, P. G. Bolhuis, and P. R. ten Wolde, "Combining molecular dynamics with mesoscopic Green's function reaction dynamics simulations," The Journal of Chemical Physics, vol. 143, no. 21, p. 214102, 2015-12.

[28] A. Vijaykumar, T. E. Ouldridge, P. R. ten Wolde, and P. G. Bolhuis, "Multiscale simulations of anisotropic particles combining molecular dynamics and Green's function reaction dynamics," The Journal of Chemical Physics, vol. 146, no. 11, p. 114106, 2017-03.

[29] M. Dibak, M. J. del Razo, D. De Sancho, C. Schütte, and F. Noé, "MSM/RD: Coupling Markov state models of molecular kinetics with reaction-diffusion simulations," The Journal of Chemical Physics, vol. 148, no. 21, p. 214107, 2018-06.

[30] L. Sbailò and F. Noé, "An efficient multi-scale Green's function reaction dynamics scheme," Journal of Chemical Physics, vol. 147, no. 18, 2017.

[31] M. E. Johnson and G. Hummer, "Free-Propagator Reweighting Integrator for Single-Particle Dynamics in Reaction-Diffusion Models of Heterogeneous Protein-Protein Interaction Systems," Physical Review X, vol. 4, no. 3, p. 031037, 2014-09.

[32] D. E. Scott, A. R. Bayly, C. Abell, and J. Skidmore, "Small molecules, big targets: Drug discovery faces the protein-protein interaction challenge," Nature Reviews Drug Discovery, vol. 15, no. 8, pp. 533-550, 2016.

[33] F. Paul, C. Wehmeyer, E. T. Abualrous, H. Wu, M. D. Crabtree, J. Schöneberg, J. Clarke, C. Freund, T. R. Weikl, and F. Noé, "Protein-peptide association kinetics beyond the seconds timescale from atomistic simulations," Nature Communications, vol. 8, no. 1, p. 1095, 2017-12.

[34] N. Plattner, S. Doerr, G. De Fabritiis, and F. Noé, "Complete protein-protein association kinetics in atomic detail revealed by molecular dynamics simulations and Markov modelling," Nature Chemistry, vol. 9, no. 10, pp. 1005-1011, 2017-06.

[35] M. Armand and J. M. Tarascon, "Building better batteries," Nature, vol. 451, no. 7179, pp. 652-657, 2008.

[36] S. Boyanov, J. Bernardi, F. Gillot, L. Dupont, M. Womes, J.-M. Tarascon, L. Monconduit, and M.-L. Doublet, "FeP: Another Attractive Anode for the Li-Ion Battery Enlisting a Reversible Two-Step Insertion/Conversion Process," Chemistry of Materials, vol. 18, no. 15, pp. 3531-3538, 2006-07. 
[37] M. von Smoluchowski, "Versuch einer mathematischen Theorie der Koagulationskinetik kolloider Lösungen," Z. Phys. Chem., no. 92, p. 129, 1916.

[38] F. C. Collins and G. E. Kimball, "Diffusion-controlled reaction rates," J. Colloid Sci., vol. 4, no. 4, pp. 425-437, 1949.

[39] M. J. del Razo and H. Qian, "A discrete stochastic formulation for reversible bimolecular reactions via diffusion encounter," Communications in Mathematical Sciences, vol. 14, no. 6, pp. 1741-1772, 2016.

[40] N. Agmon and A. Szabo, "Theory of reversible diffusion-influenced reactions," The Journal of Chemical Physics, vol. 92, no. 9, p. 5270, 1990.

[41] E. Teramoto and N. Shigesada, "Theory of Bimolecular Reaction Processes in Liquids," Progress of Theoretical Physics, vol. 37, no. 1, pp. 29-51, 1967-01.

[42] M. Doi, "Stochastic theory of diffusion-controlled reaction," Journal of Physics A: Mathematical and General, vol. 9, no. 9, p. 1479, 1976.

[43] W. Du and P. G. Bolhuis, "Sampling the equilibrium kinetic network of Trp-cage in explicit solvent," The Journal of Chemical Physics, vol. 140, no. 19, p. 195102, 2014-05.

[44] M. Doi, "Theory of diffusion-controlled reactions between non-simple molecules. I," Chemical Physics, vol. ll, pp. 107-113, 1975.

[45] M. Dibak, C. Fröhner, F. Höfling, and F. Noé, "Diffusion influenced reaction rates in the presence of a pair potential," in preparation.

[46] N. G. Van Kampen, Stochastic processes in physics and chemistry. Elsevier, 1 ed., 1992.

[47] M. J. Morelli and P. R. ten Wolde, "Reaction Brownian dynamics and the effect of spatial fluctuations on the gain of a push-pull network," The Journal of Chemical Physics, vol. 129, no. 5, p. 054112, 2008.

[48] H. C. R. Klein and U. S. Schwarz, "Studying protein assembly with reversible Brownian dynamics of patchy particles," The Journal of Chemical Physics, vol. 140, no. 18, p. 184112, 2014-05.

[49] A. Donev, C.-y. Yang, and C. Kim, "Efficient reactive Brownian dynamics," The Journal of Chemical Physics, vol. 148, no. 3, p. 034103, 2018-01.

[50] N. Metropolis, A. W. Rosenbluth, M. N. Rosenbluth, A. H. Teller, and E. Teller, "Equation of State Calculations by Fast Computing Machines," The Journal of Chemical Physics, vol. 21, no. 1953, pp. 1087-1092, 1953.

[51] W. K. Hastings, "Monte carlo sampling methods using Markov chains and their applications," Biometrika, vol. 57, no. 1, pp. 97-109, 1970.

[52] P. Atkins and J. de Paula, Atkins' physical chemistry. Oxford: Oxford University Press, 8 ed., 2006.

[53] M. P. Allen and D. J. Tildesley, Computer Simulation of Liquids. New York: Oxford University Press, 1987.

[54] C. Nuoffer and W. E. Balch, "GTPases: multifunctional molecular switches regulating vesicular traffic.," Annual review of biochemistry, vol. 63, pp. 949-90, 1994.

[55] A. Hall and C. D. Nobes, "Rho gtpases: molecular switches that control the organization and dynamics of the actin cytoskeleton," Philosophical Transactions of the Royal Society of London B: Biological Sciences, vol. 355, no. 1399, pp. 965-970, 2000.

[56] A. L. Marat, A. Wallroth, W. T. Lo, R. Müller, G. D. Norata, M. Falasca, C. Schultz, and V. Haucke, "mTORC1 activity repression by late endosomal phosphatidylinositol 3,4bisphosphate," Science, vol. 356, no. 6341, pp. 968-972, 2017.

[57] J. Lippincott-Schwartz, "Development and Use of Fluorescent Protein Markers in Living Cells," Science, vol. 300, pp. 87-91, apr 2003. 


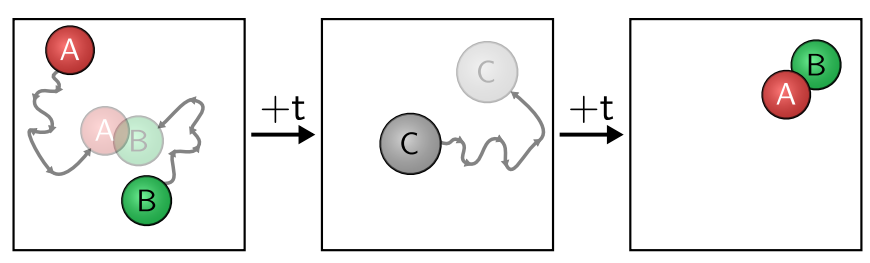

Figure 1. Schematic time evolution of a reaction-diffusion system of an isolated pair subject to the reaction $\mathrm{A}+\mathrm{B} \rightleftarrows \mathrm{C}$ with the Doi model. Particles $\mathrm{A}$ and $\mathrm{B}$ diffuse and can form a complex particle $\mathrm{C}$ when they are closer than a certain reaction radius, here depicted as the sum of the radii of the two particles. The complex particle $\mathrm{C}$ diffuses as well and can dissociate into $\mathrm{A}$ and $\mathrm{B}$ again.

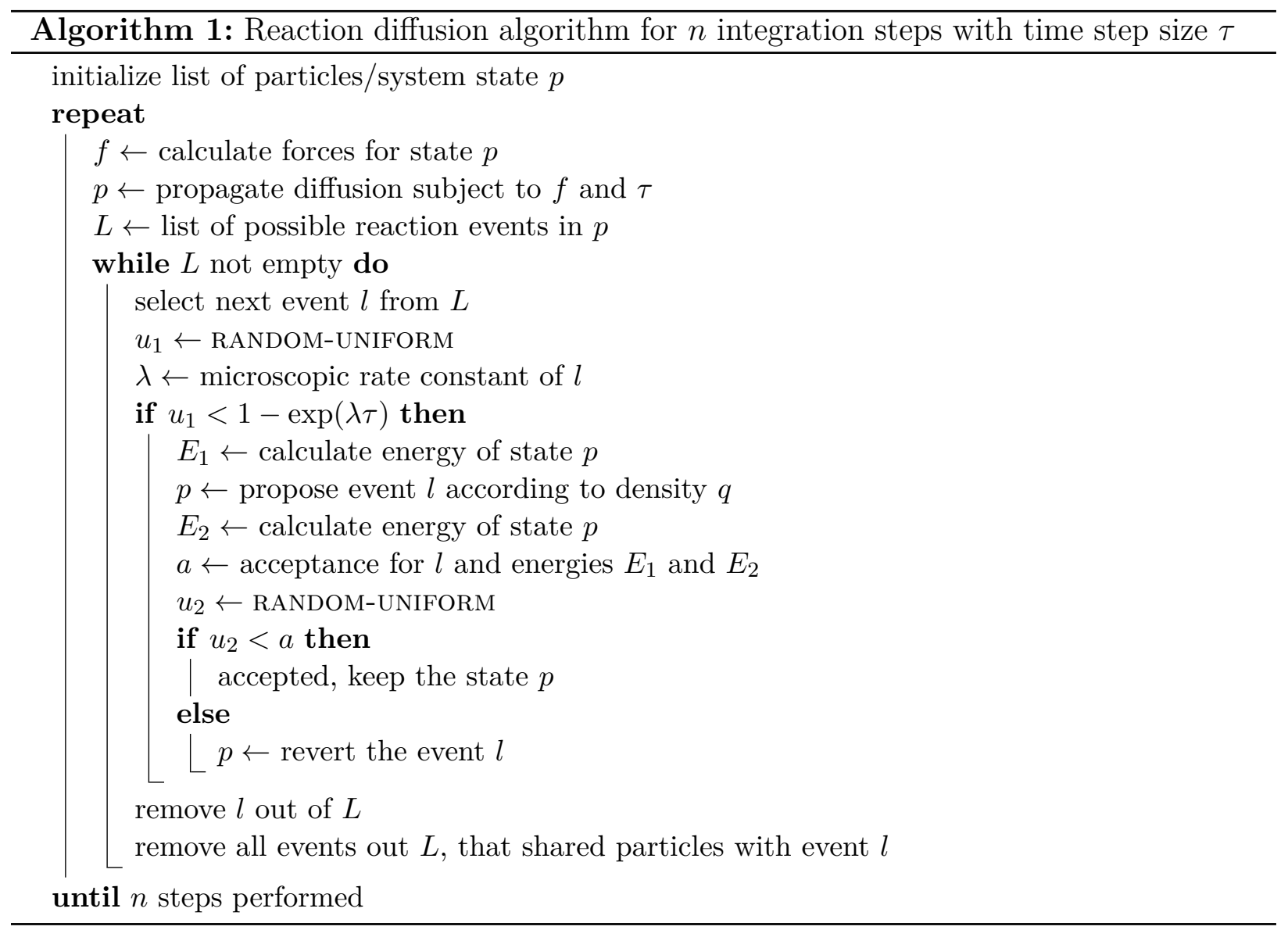


(a)
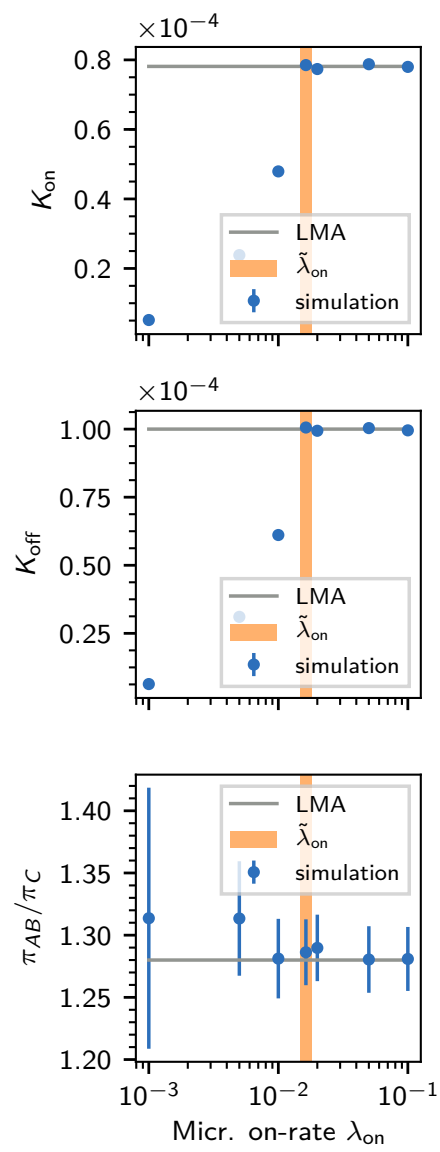

(b)
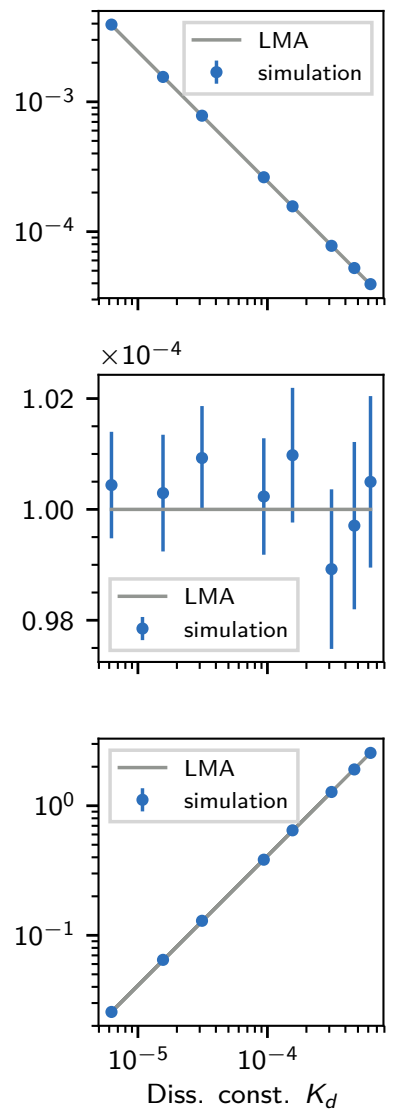

Figure 2. Validation of the proposed detailed balance reaction scheme in dilute systems by stochastic particle-based reaction-diffusion simulations (see Alg. 1). Shown are observables of the macroscopic reaction kinetics: the effective association rate $K_{\text {on }}$, the effective dissociation rate $K_{\text {off }}$ and the equilibrium constant $\pi_{A B} / \pi_{C}$. Reference values (law of mass action - LMA) for $K_{\text {on }}, K_{\text {off }}$ and $\pi_{A B} / \pi_{C}$ correspond to macroscopic behaviour described in Sec. II. See simulation parameters in Tab. I. (a) Microscopic association rate constant $\lambda_{\text {on }}$ is varied. $\tilde{\lambda}_{\text {on }}$ corresponds to Eq. (19). (b) The given dissociation constant $K_{d}$ is varied. The microscopic association rate constant is $\lambda_{\text {on }}=\tilde{\lambda}_{\text {on }}\left(K_{d}\right)$. 


\begin{tabular}{lcc}
\hline Quantity & Symbol & Value \\
\hline Dissociation constant & $K_{d}$ & $3.125 \times 10^{-4}$ \\
Dissociation rate constant & $k_{\text {off }}$ & $10^{-4}$ \\
Volume & $V$ & $16 \times 16 \times 16$ \\
Diffusion constant of each particle & $D$ & 5 \\
Reaction radius & $R_{\text {reac }}$ & 2 \\
Interaction radius & $R_{\text {int }}$ & 2 \\
Force constant & $\kappa$ & 5 \\
Time step length & & \\
in Fig. 2 & $\tau_{1}$ & $10^{-4}$ \\
$\quad$ in Fig. 3 & $\tau_{2}$ & $1.25 \times 10^{-5}$ \\
Number of integration steps & & \\
in Fig. 2 & $m_{1}$ & $3 \times 10^{10}$ \\
in Fig. 3 & $m_{2}$ & $4.8 \times 10^{11}$ \\
\hline
\end{tabular}

Table I. Unitless parameters used in the simulations of dilute systems, see Fig. 2 and 3.

(a) Doi reaction scheme

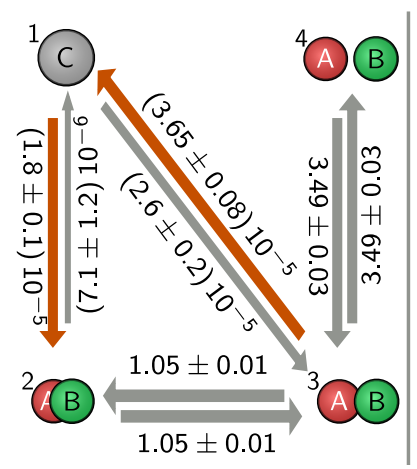

(b) Proposed DB reaction scheme

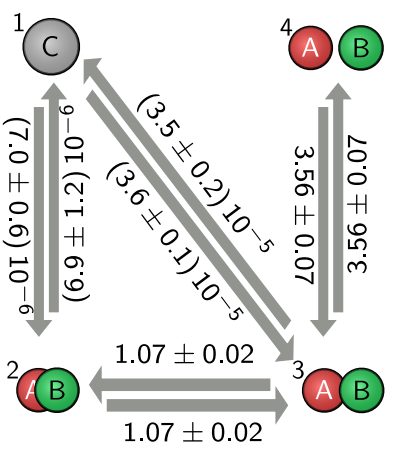

Figure 3. Probability fluxes between associated and dissociated states measured from particle-based reaction-diffusion simulations (see Alg. 1) in the dilute limit. Compared are the Doi reaction scheme and the proposed detailed balance reaction scheme (DB). Definitions of the states 1-4 are given in Sec. IV A 2. Arrows depict transitions between these states as observed in the simulations. The width of the arrows encodes the probability flux $\pi_{i} K_{i j}$, also given as numeric values measured from multiple independent simulations giving rise to the standard error of the mean. The widths of two adjacent arrows are normalized with respect to each other (not globally). See parameters in Tab. I. (a) Doi reaction scheme. The probability fluxes for the transitions $1 \rightarrow 2$ and $1 \rightarrow 3$ are imbalanced compared to their respective counterparts, resulting in a circular flux of probability. (b) Detailed balance reaction scheme. 


\begin{tabular}{lcc}
\hline Quantity & Symbol & Value \\
\hline Dissociation constant & $K_{d}$ & $2 \times 10^{-2}$ \\
Dissociation rate constant & $k_{\text {off }}$ & $10^{-3}$ \\
Volume & $V$ & $20 \times 20 \times 20$ \\
Particle radii & & \\
$\quad$ case $r_{A}^{3}+r_{B}^{3}<r_{C}^{3}$ & $\left(r_{A}, r_{B}, r_{C}\right)$ & $(1,1,1.4)$ \\
$\quad$ case $r_{A}^{3}+r_{B}^{3}>r_{C}^{3}$ & $\left(r_{A}, r_{B}, r_{C}\right)$ & $(1,1,1.1)$ \\
Diffusion constants per radius & & \\
for species $i \in\{A, B, C\}$ & $D / r_{i}$ & 5 \\
Interaction radius for pair & & \\
$\quad$ of species $(i, j) \forall i, j \in\{A, B, C\}$ & $R_{\text {int }}(i, j)$ & $r_{i}+r_{j}$ \\
Reaction radius & $R_{\text {reac }}$ & 2 \\
Force constant & $\kappa$ & 10 \\
Time step length & $\tau$ & $5 \times 10^{-4}$ \\
Time steps until equilibrated & & \\
dilute system with $n=50$ & $m_{\text {dilute }}$ & $1.2 \times 10^{8}$ \\
dense system with $n=900$ & $m_{\text {dense }}$ & $9 \times 10^{6}$ \\
\hline
\end{tabular}

Table II. Unitless parameters used in the simulations of dense systems, see Fig. 4 and 5.

\begin{tabular}{|c|c|c|c|}
\hline & $\underbrace{\mathrm{A}+\mathrm{B}}_{\mathbf{x}} \underbrace{\stackrel{k_{\mathrm{on}}}{\rightleftarrows}}_{k_{\mathrm{off}}} \underbrace{\mathrm{C}}_{\mathbf{y}}$ & $\underbrace{A}_{\mathbf{x}} \underbrace{\stackrel{k_{\text {on }}}{\rightleftarrows}}_{k_{\text {off }}} \underbrace{\mathrm{B}}_{\mathbf{y}}$ & 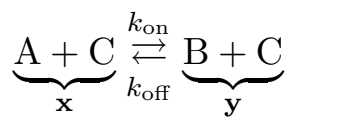 \\
\hline$\lambda^{+}(\mathbf{x})$ & $\lambda_{\text {on }} \chi_{\text {reac }}(\mathbf{x})$ & $\lambda_{\text {on }}$ & $\lambda_{\text {on }} \chi_{\text {reac }}(\mathbf{x})$ \\
\hline$\lambda^{-}(\mathbf{y})$ & $\lambda_{\text {off }}$ & $\lambda_{\text {off }}$ & $\lambda_{\text {off }} \chi_{\text {reac }}(\mathbf{y})$ \\
\hline$q^{+}(\mathbf{y} \mid \mathbf{x})$ & $V^{-2} \delta\left(\mathbf{y}_{c}-\frac{\mathbf{x}_{a}+\mathbf{x}_{b}}{2}\right)$ & $\delta(\mathbf{y}-\mathbf{x})$ & $\delta(\mathbf{y}-\mathbf{x})$ \\
\hline$q^{-}(\mathbf{x} \mid \mathbf{y})$ & $\left(V V_{\text {reac }}^{\text {eff }}\right)^{-1} \delta\left(\mathbf{y}_{c}-\frac{\mathbf{x}_{a}+\mathbf{x}_{b}}{2}\right)$ & $\delta(\mathbf{x}-\mathbf{y})$ & $\delta(\mathbf{x}-\mathbf{y})$ \\
\hline$f^{+}(\mathbf{y} \mid \mathbf{x})$ & $\begin{array}{c}\ldots \times \chi_{\mathrm{reac}}(\mathbf{x}) e^{-\beta U_{A B}(\mathbf{x})} \\
e^{-\beta\left(E(\mathbf{y})-\left[E(\mathbf{x})-U_{A B}(\mathbf{x})\right]\right)}\end{array}$ & $e^{-\beta(E(\mathbf{y})-E(\mathbf{x}))}$ & $\frac{V_{\text {reac, }}^{\text {eff }}}{V_{\text {reac }}^{\text {eff }}} e^{-\beta(E(\mathbf{y})-E(\mathbf{x}))}$ \\
\hline$f^{-}(\mathbf{x} \mid \mathbf{y})$ & $e^{-\beta\left(\left[E(\mathbf{x})-U_{A B}(\mathbf{x})\right]-E(\mathbf{y})\right)}$ & $e^{-\beta(E(\mathbf{x})-E(\mathbf{y}))}$ & $\frac{V_{\text {reac, } \mathrm{B}}^{\text {eff }}}{V_{\text {reac, } \mathrm{A}}^{\text {eff }}} e^{-\beta(E(\mathbf{x})-E(\mathbf{y}))}$ \\
\hline constraints & $\begin{array}{l}k_{\mathrm{on}}=\lambda_{\mathrm{on}} V \frac{V_{\mathrm{reac}}^{\mathrm{eff}}}{V-V_{\mathrm{ex}}} \\
k_{\mathrm{off}}=\lambda_{\mathrm{off}}\end{array}$ & $\begin{array}{l}k_{\mathrm{on}}=\lambda_{\mathrm{on}} \\
k_{\mathrm{off}}=\lambda_{\mathrm{off}}\end{array}$ & $\begin{array}{l}k_{\mathrm{on}}=\lambda_{\mathrm{on}} V \frac{V_{\mathrm{reac}, \mathrm{A}}^{\mathrm{eff}}}{V-V_{\mathrm{ex}, \mathrm{A}}} \\
k_{\mathrm{off}}=\lambda_{\mathrm{off}} V \frac{V_{\text {reac, }}^{\text {eff }}}{V-V_{\mathrm{ex}, \mathrm{B}}}\end{array}$ \\
\hline
\end{tabular}

Table III. Summary of the iPRD-DB quantities for three different kinds of reversible reactions: reversible association (see Sec. II B), reversible unimolecular conversion, and reversible bimolecular enzymatic reaction (see Sec. III C). Quantities are: absolute proposal rates $\lambda$, proposal densities $q$, and acceptance probabilities $\alpha=\min \{1, f\}$, as described in Sec. III. Superscript + and - denote the "on" and "off" process respectively, corresponding to the definition of the reaction. $\mathbf{x}$ and $\mathbf{y}$ are the microscopic positions of particles. Constraints describe for which microscopic parameters the acceptance probabilities will be unity in the dilute limit. 
(a)
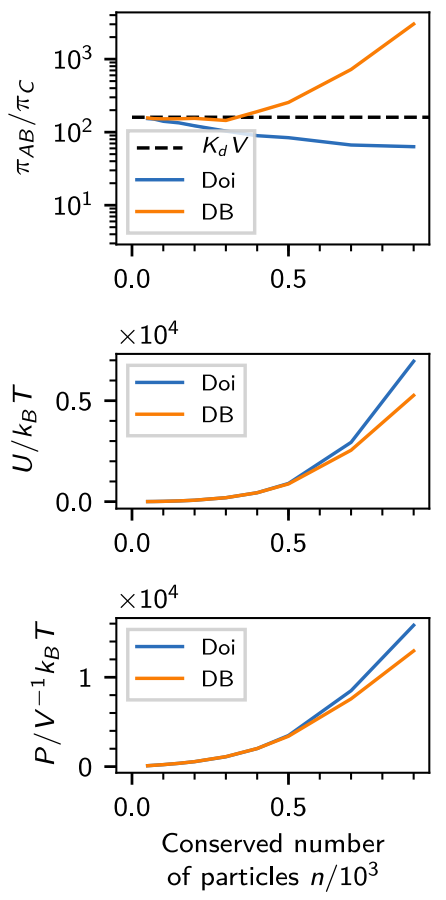

(b) $(A)+B>$ (C)
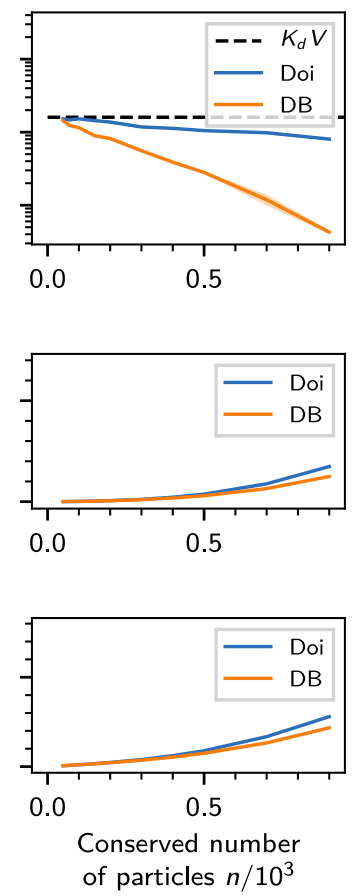

Figure 4. Steady state observables measured in particle-based reaction-diffusion simulations with multiple particles. The quantity $n=\left(N_{A}+N_{B}\right) / 2+N_{C}$ is conserved during a simulation. Shown are ensemble- and time-averaged values of the equilibrium constant $\pi_{A B} / \pi_{C}=V[A][B] /[C]$, the potential energy $U$ in units of $k_{B} T$, the pressure $P$ in units of $V^{-1} k_{B} T$. Compared are the two reaction schemes Doi and DB, see Sec. IV. See simulation parameters in Tab. II (a) An association reaction of $\mathrm{A}$ and $\mathrm{B}$ increases the total volume occupied by particles such that $r_{A}^{3}+r_{B}^{3}<r_{C}^{3}$. (b) The $\mathrm{C}$ particle occupies less volume than A and B combined such that $r_{A}^{3}+r_{B}^{3}>r_{C}^{3}$ 


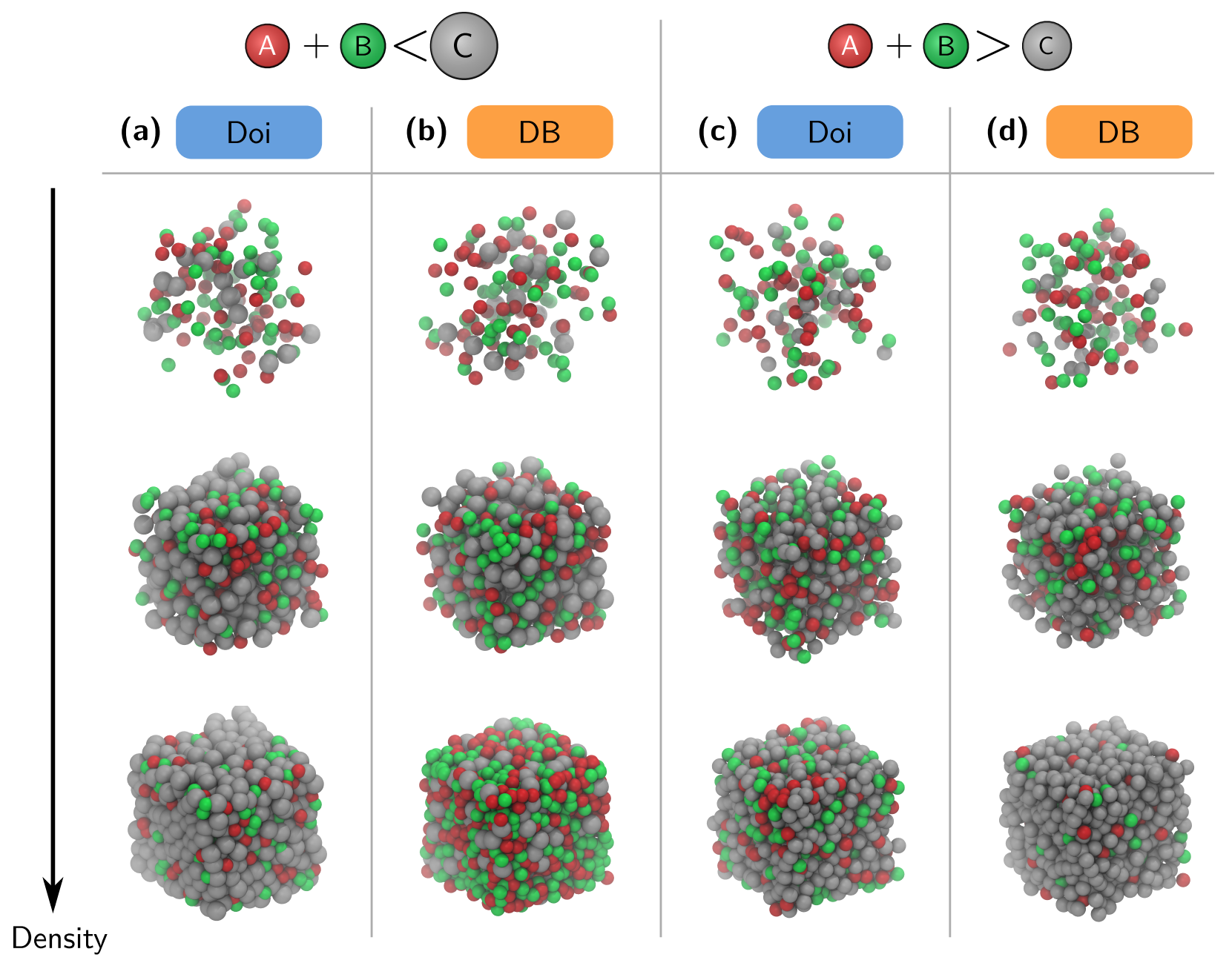

Figure 5. Steady state configurations of particle-based reaction-diffusion simulations subject to the reaction $\mathrm{A}+\mathrm{B} \rightleftarrows \mathrm{C}$ for different densities in terms of the number of particles $n$ initially in the system. Compared are the two reaction schemes Doi and DB, see Sec. IV at different particle radii respectively. See simulation parameters in Tab. II. (a) The associated state occupies more volume than the dissociated state, reactions are handled with the Doi scheme. (b) The associated state occupies more volume than the dissociated state, reactions are handled with the DB scheme (c) The associated state occupies less volume than the dissociated state, reactions are handled with the Doi scheme. (d) The associated state occupies less volume than the dissociated state, reactions are handled with the DB scheme. 